\title{
Computational Fluid Dynamics Framework for Large-Scale Simulation in Pediatric Cardiology
}

\author{
Kristóf Ralovich ${ }^{1}$, Razvan Ionasec ${ }^{2}$, Viorel Mihalef ${ }^{2}$, Puneet Sharma ${ }^{2}$, Bogdan \\ Georgescu $^{2}$, Allen Everett ${ }^{3}$, Nassir Navab ${ }^{1}$, and Dorin Comaniciu ${ }^{2}$ \\ 1 Technical University of Munich, Boltzmannstr. 3, 85748 Garching, Germany \\ 2 Siemens Corporate Research, Princeton, NJ 08540, USA \\ 3 Johns Hopkins University, Baltimore, MD 21287, USA
}

\begin{abstract}
There is a high demand for patient specific cardiovascular therapeutics, especially in pediatric cardiology which is confronted with complex and rather unique congenital diseases. Current predictors for disease severity and treatment selection have been proven to be suboptimal creating profound burden of premature morbidity and mortality. Over the past decade, the influence of blood hemodynamics has become increasingly acknowledged, especially in the context of congenital diseases of the aortic arch. MRI-based 2D and 3D flow measurements are nowadays possible, although restricted by cumbersome acquisition protocols and limited acquisition resolution. Computational fluid dynamics offers a valuable alternative that also enables treatment outcome prediction. However, the current methods rely on a sequence of complicated manual steps that lack the scalability required within clinical settings. We propose a computation framework for large-scale hemodynamics simulations in pediatric cardiology to aid diagnostic and therapy decision making in patients affected by congenital disease of the aortic valve and the aorta. Our method provides a deterministic and streamlined processing pipeline to perform computational fluid dynamics simulations based on patient-specific boundary conditions. Thus, blood flow simulations are performed using an embedded boundary method within a level-set formulation with boundary conditions provided by patient-specific anatomical and hemodynamical models. The capabilities of our framework are demonstrated by performing blood flow analysis on patients selected from a FDA sponsored multi-center clinical trial.
\end{abstract}

\section{Introduction}

There is a high demand for patient specific cardiovascular therapeutics, especially in pediatric cardiology which is confronted with complex and rather unique congenital diseases. The incidence of congenital heart disease (CHD) is about 75/1, 000 live births, out of which dysfunctions of the aortic valve and the aorta is a major subgroup that requires expert cardiologic care [6]. Aortic diseases are rarely isolated disorders but rather occur in presentments of other birth defects. For instance $50 \%$ of patients with aortic coarctation have a bicuspid aortic valve, 
which is also common in non-genetic aortic aneurysms. Marfan's patients, in addition to aortic aneurysms, typically have both functionally-abnormal aortic and mitral valves as a consequence of their fibrillin gene defect. Without appropriate clinical management the wall of the aorta can dissect or even rupture, which leaves little hope for survival and usually results in sudden death.

In case of aortic aneurysm the clinical state of the art is based on prompt diagnosis of risk factors and subsequent onset of therapy as soon as the diameter of the ascending aorta has reached a predefined value [20]. However, aortic diameter is an imperfect predictor of aortic dissection or rupture and the current criterion of a diameter $>50 \mathrm{~mm}$ misses $40 \%$ of dissections. Therefore, even today, the burden of premature morbidity and mortality is profound. In contrast to aortic aneurysm, coarctation of the aorta is a common discrete or sometimes tubular congenital narrowing within the lumen of the aorta. Percutaneous balloon angioplasty and stent implantation have displaced surgery as primary therapy for native and recurrent coarctation [17]. The state of the art is balloon or stent enlargement of the aorta no larger than the surrounding non-diseased aorta. However this approach is commonly associated with the development of early and late aortic dissection and aneurysm formation or suboptimal clinical improvement [3]. Presently no guidance exists to personalize percutaneous therapy to achieve an optimal therapeutic outcome with the least initial and long term risk for the patient.

The influence of blood hemodynamics on disease progression and therapeutical outcome has become increasingly acknowledged [15]. Over the past two decades methods for blood flow measurements using 2D time-resolved (Cine) phase contrast (PC) MRI have continuously evolved [13]. Newest MRI systems enable today 3D Cine PC-MRI with three-directional flow encoding that can capture blood velocities within a 3D volume of interest [10]. Using this technique [4], significant variations in flow patterns where observed even for mild aortic pathologies. Nevertheless, 3D flow measurements are limited in resolution, and cumbersome and time consuming to acquire even in research settings. In addition, in vivo flow analysis does not enable for the simulation and prediction of hemodynamic performance related to specific cardiac procedures.

The potential of advanced disease analysis and therapy planning systems based of computational fluid dynamics (CFD) has been illustrated in [18]. The optimal treatment option for a Fontan patient with severe left pulmonary arteriovenous malformation was determined by simulating multiple surgical scenarios with the associated predicted hemodynamic parameters. The role of CFD, coupled with patient-specific inflow conditions, for treatment decisions of aortic pathologies was presented in [7]. In [8] hemodynamics simulations are investigated in the context of coronary artery disease management. The major limitation of previously published methods is the lack of scalability. Approaches are customized for a limited number of well chosen patients (usually 1 - 3) and involve a cascade of tedious manual processing tasks, which result in hardly reproducible results. 
We propose a unified computational framework for large-scale hemodynamic modeling and simulations in pediatric cardiology to aid diagnostic and therapy decision making in patients affected by congenital disease of the aortic valve and the aorta. Our method provides a deterministic and streamlined processing pipeline to perform Computational Fluid Dynamics (CFD) simulations from patient-specific data. The proposed method includes an automated approach to segment the centerline and lumen of the aorta as well as the inlet and outlet flow profiles over the entire cardiac cycle. CFD simulations are performed using an embedded boundary method solved within a level-set formulation. We demonstrate the capabilities of our framework by performing blood flow simulations on selected patients coming from a FDA sponsored multi-center clinical trial, Coarctation Of the Aorta Stent Trial (COAST). To this end, we present flow pattern analysis in three patients that suffer from aortic coarctation and various types of aortic valve dysfunctions.

\section{Description of method}

Within this section we describe in detail each component of the proposed simulation framework as is illustrated in Figure 1. The integrated steps are: 1) Selection and reconstruction of MR measurements of anatomy and flow (Sec. 2.1), 2) Estimation of the Patient-Specific Aorta Anatomy (Sec. 2.2) 3) Estimation of the Patient-Specific Aorta Flow (Sec. 2.3), 4) Computational Fluid Dynamic Simulation.
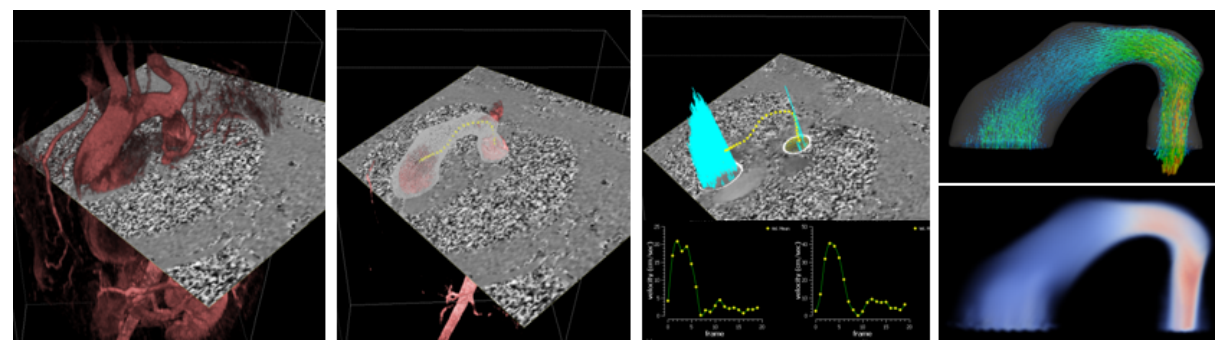

Fig. 1. Pipeline of the simulation: (a) Volume rendering of a clear, high contrast CEMRA image displayed together with a PC-MRI slice used for depicting an aortic arch. (b) Extracted centerline and segmentation of the aorta (c) Patient specific systolic in- and outflow rates derived from PC-MRI measurement. (d) Simulated blood flow velocities and vorticity magnitude.

\subsection{Measurements of Anatomy and Flow}

The CFD simulations within our framework are driven by patient-specific measurements coming from MR images. We would like to emphasize that we directly 
process raw DICOM images produced by MR scanner of all major vendors. From a typical study that contains on avg. 1500 2D images we utilize two sequences: 1 ) A stack of contrast enhanced MR angiography (CE-MRA) slices to reconstruct the 3D anatomy of the aorta 2) A 2D phase contrast (PC) -MRI Cine sequence that contains through-plane flow velocity information distal to the aortic valve and the region of the descending aorta, distal to the supra-aortic arteries. The reconstruction and image selection is accomplished within approx. 3 minutes (Figure 1(a)).

The 3D CE-MRA images are coronal stacks of dimension $256 \times 256$ to $512 \times 640$ with $56-140$ slices, in-plane resolution isotropic $0.605-1.562 \mathrm{~mm}$, slice thickness of $0.889-1.8 \mathrm{~mm}$. The coronal images usually contain the left ventricular outflow tract (LVOT), the aortic valve (AV), the aortic arch, including the 3 superior arteries, and the descending thoratic aorta. Due to the length of the acquisition, the angiograms correspond to the aortic lumen motion averaged over the duration of the scan.

Flow sensitive ECG gated Cine PC MR images are typically oblique axial time-series encoding through-plane velocities in the isotropic resolution of $0.742-2.083 \mathrm{~mm}$, dimension $126 \times 144$ to $384 \times 512$, VENC found in the range of $140-300 \mathrm{~cm} / \mathrm{s}$. The slices are routinely positioned to provide two different cross-sections, one somewhere between the LVOT and ascending aorta (AAo), the other in the DAo (if there is a stent implant, in the direction of the blood flow below the stent location). Each time-series correspond to one heart cycle, and have 20-40 frames per cycle. The heart rates of the examined patients are ranging from $60-75 \mathrm{bm}$. All patient data was acquired employing 1.5 Tesla scanners as part of existing clinical practice, and not specifically for this simulation study.

\subsection{Estimation of the Patient-Specific Aorta Anatomy}

Aorta Centerline Extraction Within this step the centerline of the aorta is automatically extracted from the 3D anatomy image. Two seed points in the lumen of the aorta are required to initialize the algorithm, with the source seed point placed in the left ventricle outflow tract (LVOT) region and the sink seed point in the descending thoracic aorta. The automatic extraction is performed by means of a robust vessel tree modeling algorithm similarly as in [5]. The graph-based optimization algorithm estimates vessel centerlines using multi-scale medialness filters to model circular like cross-sections of vessel geometries. As illustrated in Figure 1(b) the result of the centerline extraction is an ordered set of points along the geometrical mean of the thoratic aortic lumen and is performed within approx. 30 seconds.

Aorta Segmentation The extracted centerline is uniformly sampled to extract orthogonal circular structures of constant radius $(r=20 \mathrm{~mm})$ that provide positive samples for a min-cut/max-flow segmentation algorithm [2]. Negative samples are generated outside at the same locations along the centerline at a radius $r=75 \mathrm{~mm}$. The segmented image is undergoing a marching cubes iso-surface [9] 
extraction to produce a polygonal mesh of the lumen geometry. Further processing of the unstructured mesh involves welding identical vertices, keeping the largest connected component with surface smoothing constraints [1] to retain a manifold triangular surface mesh. Figure 1(c) illustrates the result of the aorta segmentation performed within approx. $5 \mathrm{~min}$.

\subsection{Estimation of the Patient-Specific Aorta Flow}

Within this step the patient-specific flow profiles over the entire cardiac cycle are extracted at the aortic inflow and outflow from the 2D PC-MRI cine images. Typically, the PC-MRI sequence is easily registered with the anatomy image and aortic segmentation using the MR machine coordinates. The intersection of the PC-MRI image plane and the vessel geometry defines two 2D closed contours. These planar patches are densely triangulated and treated as an inflow and outflow profile, respectively. Inside each patch a uniform grid sampling of the PC-MRI image is performed at the pixel center locations to obtain spatially constrained velocity values over the entire cardiac cycle. It is important to note that depending on the scanner type and vendor particular care must be taken for the correct normalization of the flow velocity encoding.

\subsection{Computational Fluid Dynamics of Aortic Blood Flow}

We model the blood flow dynamics in the aorta using 3D incompressible NavierStokes equations with viscous terms - the standard continuum mechanics model for fluid flow. The equations are discretized and solved with the embedded boundary method. We use both finite difference and finite volume techniques to solve the fractional step combined with an approximate projection method for the pressure. The blood is modeled as a Newtonian fluid, which is generally accepted as a reasonable first approximation to the actual behavior of blood at shear rates observed in large arteries [14].

An important advantage of the framework we use to obtain the computational fluid dynamics (CFD) results for the aortic blood flow is its fully automatic nature. This is achieved using an Eulerian framework and embedding the boundary using a level set function, which implicitly defines the computational domain. The level set is computed as the signed-distance from the aortic triangular mesh. This is done first in the cells located near the mesh itself, then the signs are extended to the rest of the rectangular domain by solving an extrapolation equation in fictitious time similarly to [11] [12]. Note that the exact values of the level set are important for the various discretizations only near the boundary, and only its sign matters away from the mesh. This is visible in Figure 3(a). To reduce the sensitivity of the simulation results with respect to the outlet boundary conditions, and thus improve the simulation results, we extend the outlet before applying the pressure outlet boundary condition. The extension is approximately 6-8 times the radius of the outlet. 
The boundary conditions used in the simulations are as follows: at the aortic walls we use no-slip for the velocity and the appropriate normal balance (translating into a Neumann boundary condition) for the pressure. The inflow velocity is extrapolated from the MRI, using smooth kernels, to all the inlet nodes, while pressure proportional to the flow is set as a Dirichlet boundary condition on the outlet faces. The inflow velocities are also interpolated in time using second order accurate interpolation.

Our computational algorithm starts at a given time step $n$ from the velocity and pressure information at the previous time step $u^{n-1}, p^{n-1}$, and computes $u^{n}, p^{n}$ following a fractional step projection method. The geometry (hence the level set) is considered static in this paper, but the level set formulation allows for an easy and robust extension of the algorithm to moving boundaries. Our algorithm computes the solution to the unsteady 3D Navier-Stokes equations in the following steps: 1) Convective update for the velocity $u$ 2) Semi-implicit update for the velocity (viscous force contribution) 3) Pressure update by solving the pressure Poisson equation with mixed boundary conditions 4) New velocity update. The algorithm uses subcycling to enforce the Courant-Friendrichs-Levy (CFL) condition at every time step. Several results obtained with our system are described and discussed in the Experiments and validation section.

\subsection{Discussion of limitations}

The current simulations use several simplified assumptions involving the boundary conditions and the constitutive equations, which may benefit from further extensions. The geometric model is temporally fixed, which may be improved by using a moving boundaries formulation, as done for example in [11]. As underlined by [19] specifiying the correct boundary conditions is of utmost importance for the realism of the computations, e.g. for simulating the phase delay between flow and pressure. In the current formulation we can recover the inlet/outlet flow delay by setting the outlet pressure boundary conditions at the descending aorta proportional to the flow, as measured by PC-MRI on the descending aortic cross section.

A possible extension of our current setup is the inclusion of turbulence modeling. While many previous works have not included turbulence shear terms in their models, such terms may be needed due to the high Reynolds numbers (sometimes greater than 8000) achieved during systole in the ascending aorta, especially considering that we are using pathological cases which are often characterized by increased Reynolds numbers. In our framework, a first goal is to validate qualitatively the patterns obtained using CFD and the ones measured by PC-MRI. Due to the measurement location in the descending aorta being far from the regions in the ascending aorta where turbulence is generated, we chose to start with the non-turbulent model. This allows us to better quantify the influence of the aortic geometry on the flow. 


\section{Experiments and Validation}

This section presents results of hemodynamics simulations performed with the proposed method on randomly selected patients from the Coarctation of the Aorta Stent Trial (COAST) [16]. COAST is an FDA sponsored multi-center clinical trial that investigates the safety and effectiveness of Cheatham Platinum (CP) bare metal stents in the treatment of native and recurrent aortic coarctation. COAST is a multi-center trial, involving patients enrolled from multiple pediatric institutions across the US, with data acquired on MR scanners from multiple vendors by various radiologists, employing varying imaging protocols and different parameter encoding. All images used within our evaluations are acquired during follow-up studies at 12-month after transcatheter implantation of metallic stents.

Using the CFD simulation framework described above we have performed a series of simulations using the geometric constraints of the aortic meshes as static boundary conditions, and the sampled MRI-derived velocity as the inflow profiles (see Fig 2). The aortic data was randomly sampled from patients with various pathologies, including bicuspid valve, coarctations, artificial valves and stents. The full comparison of the simulations is beyond the scope of this (system) paper, and we give here an outline of several observed patterns that correlate with the various pathologies.

The blood density and dynamic viscosity used in the simulations were set to generic mean values across healthy individuals, namely $\rho=1.05 \mathrm{~g} / \mathrm{cm}^{3}$ and $\mu=4 \mathrm{mPa} \cdot \mathrm{s}$. The simulations featured grids with physical resolutions between $0.5-1.5 \mathrm{~mm}$, and were run on Windows PCs with Intel Xeon CPUs of $2.53 \mathrm{GHz}$. Two cycles were computed in each experiment, with total computation times varying between 12-18 hours. The multigrid PCG solver used to iteratively solve the pressure Poisson equation and the Poisson equation arising from the semiimplicit treatment of the viscous terms used a tolerance of $10 \mathrm{e}-7$ and usually converged in less than 40 iterations.

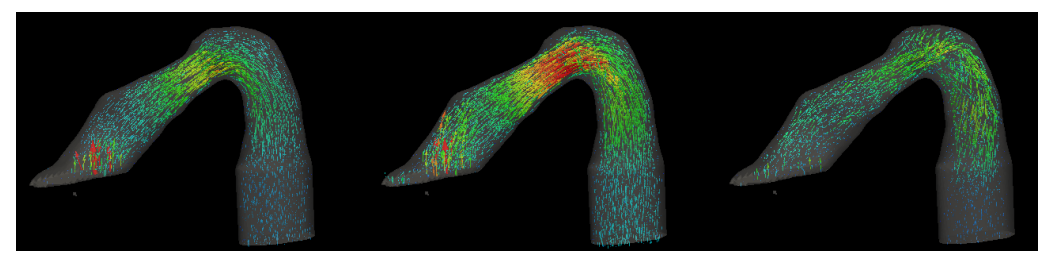

Fig. 2. Simulation sequence for systolic flow in aorta with valve replacement. Times $0.07,0.11,0.2 \mathrm{sec}$.

The cardiac cycle simulated using our CFD method features generic flow patterns like waveform delay between inlet and outlet, or increased velocities in the aortic arch (Fig. 2). Furthermore, our method also recovers patterns specific to various pathologies, as outlined below. A first experiment used aortic data 


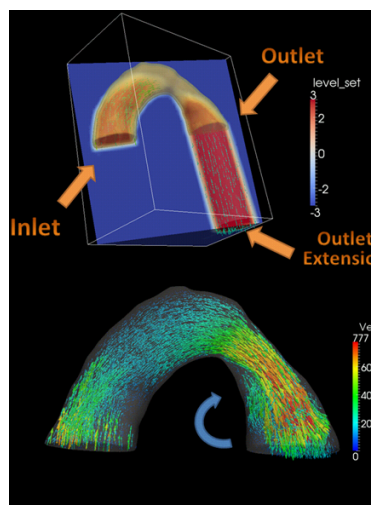

(a)

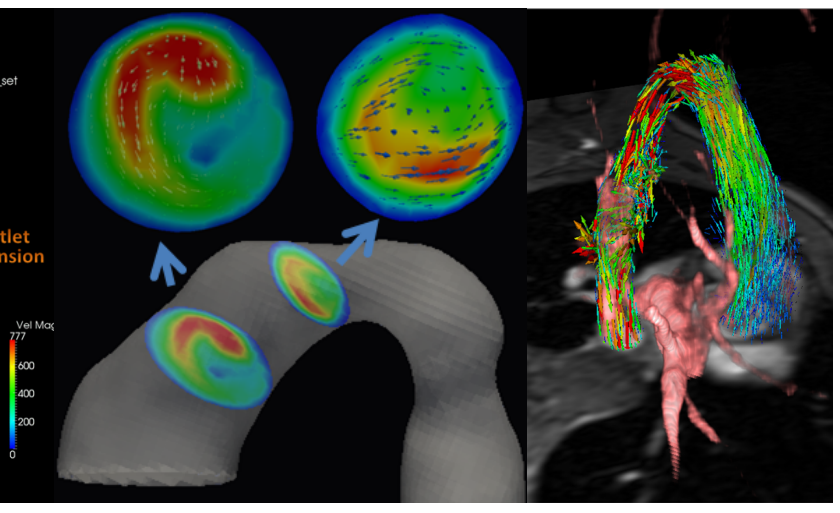

(b) (c)

Fig. 3. (a) Our computational setup: the Lagrangian aortic mesh is embedded in an Eulerian domain using a level set. Visible here are a crossection of the domain, colorcoded with the level set values, and the embedded aortic mesh (in transparent yellow) together with its outlet extension (in transparent white). The blood velocity field during early systole, simulated using CFD, is also visualized as a vector field. Below, coarctation with vortex formation. (b)Enhanced helical rotation due to bicuspid valve. (c)Simulation results overlayed with anatomical images.

featuring medium coarctation in the descending region. The vortex formation pattern specific to coarctation was observed, and is shown in Figure 3(a). A bicuspid heart experiment $3(\mathrm{~b})$ produced the enhanced helical pattern observed in such hearts, due to the blood jet that hits the aortic wall in the lower ascending aorta.

\section{Conclusion}

In the paper we presented a novel framework that enables large-scale hemodynamic modeling and simulations in pediatric cardiology to aid diagnostic and therapy decision making in patients affected by congenital disease of the aortic valve and the aorta. The streamlined processing pipeline includes an automated approach to segment the centerline and lumen of the aorta as well as the inlet and outlet flow profiles over the entire cardiac cycle. CFD simulations are performed using an embedded boundary method solved within a level-set formulation and patient-specific anatomical and hemodynamical boundary conditions. We demonstrate the capabilities of our framework by performing blood flow simulations on selected patients coming from a FDA sponsored multi-center clinical trial, Coarctation Of the Aorta Stent Trial (COAST). The simulations are shown to recover flow patterns specific to the corresponding pathologies. 


\section{Acknowledgements}

This work has been partially funded by European Union project Sim-e-Child (FP7 - 248421).

\section{References}

1. Bischoff, B.S., Botsch, M., Steinberg, S., Bischoff, S., Kobbelt, L., Aachen, R.: Openmesh - a generic and efficient polygon mesh data structure. In: In OpenSG Symposium (2002)

2. Boykov, Y., Kolmogorov, V.: An experimental comparison of min-cut/max- flow algorithms for energy minimization in vision. Pattern Analysis and Machine Intelligence, IEEE Transactions on 26(9), 1124 -1137 (2004)

3. Ewert, P., Abdul-Khaliq, H., Peters, B., Nagdyman, N., Schubert, S., Lange, P.E.: Transcatheter therapy of long extreme subatretic aortic coarctations with covered stents. Catheterization and Cardiovascular Interventions 63(2)

4. Frydrychowicz, A., Weigang, E., Harloff, A., Beyersdorf, F., Hennig, J., Langer, M., Markl, M.: Time-resolved 3-dimensional magnetic resonance velocity mapping at $3 \mathrm{t}$ reveals drastic changes in flow patterns in a partially thrombosed aortic arch. Circulation 113(11), e460-461 (2006)

5. Gülsün, M.A., Tek, H.: Robust vessel tree modeling. In: Proceedings of the 11th international conference on Medical Image Computing and Computer-Assisted Intervention - Part I. pp. 602-611. MICCAI '08 (2008)

6. Hoffman, J.I.E., Kaplan, S.: The incidence of congenital heart disease. J Am Coll Cardiol 39(12), 1890-1900 (2002)

7. Karmonik, C., Bismuth, J.X., Davies, M.G., Lumsden, A.B.: Computational hemodynamics in the human aorta: A computational fluid dynamics study of three cases with patient-specific geometries and inflow rates. Technol. Health Care 16, 343-354 (October 2008)

8. Kim, H., Vignon-Clementel, I., Figueroa, C., Jansen, K., Taylor, C.: Developing computational methods for three-dimensional finite element simulations of coronary blood flow. Finite Elements in Analysis and Design 46(6), 514 - 525 (2010), the Twenty-First Annual Robert J. Melosh Competition

9. Lorensen, W.E., Cline, H.E.: Marching cubes: A high resolution 3d surface construction algorithm. pp. 163-169. SIGGRAPH '87 (1987)

10. Markl, M., Kilner, P., Ebbers, T.: Comprehensive 4d velocity mapping of the heart and great vessels by cardiovascular magnetic resonance. Journal of Cardiovascular Magnetic Resonance 13(1), 7 (2011)

11. Mihalef, V., Ionasec, R., Wang, Y., Zheng, Y., Georgescu, B., Comaniciu, D.: Patient-specific modeling of left heart anatomy, dynamics and hemodynamics from high resolution 4d ct. In: Proceedings of the 2010 IEEE international conference on Biomedical imaging: from nano to Macro. pp. 504-507. ISBI'10 (2010)

12. Mihalef, V., Metaxas, D., Sussman, M., Hurmusiadis, V., Axel, L.: Atrioventricular blood flow simulation based on patient-specific data. In: Proceedings of the 5th International Conference on Functional Imaging and Modeling of the Heart. pp. 386-395. FIMH '09 (2009)

13. Pelc, N.J., Herfkens, R.J., Shimakawa, A., Enzmann, D.R.: Phase contrast cine magnetic resonance imaging. Magnetic Resonance Quarterly (4), 229-254 (1991) 
14. Perktold, K., Peter, R., Resch, M., Langs, G.: Pulsatile non-newtonian blood flow in three-dimensional carotid bifurcation models: a numerical study of flow phenomena under different bifurcation angles. Journal of Biomedical Engineering 13(6) (1991)

15. Richter, Y., Edelman, E.R.: Cardiology is flow. Circulation 113(23), 2679-2682 (2006)

16. Ringel, R.E., Jenkins, K.: Coarctation of the aorta stent trial (coast) (2007), http://clinicaltrials.gov/ct2/show/NCT00552812

17. Shaddy, R., Boucek, M., Sturtevant, J., Ruttenberg, H., Jaffe, R., Tani, L., Judd, V., Veasy, L., McGough, E., Orsmond, G.: Comparison of angioplasty and surgery for unoperated coarctation of the aorta. Circulation 87(3), 793-799 (1993)

18. Sundareswaran, K.S., de Zelicourt, D., Sharma, S., Kanter, K.R., Spray, T.L., Rossignac, J., Sotiropoulos, F., Fogel, M.A., Yoganathan, A.P.: Correction of pulmonary arteriovenous malformation using image-based surgical planning. J Am Coll Cardiol Img 2(8), 1024-1030 (2009)

19. Wan, J., Steele, B., Spicer, S.A., Strohband, S., Feijo, G.R., Hughes, T.J.R., Taylor, C.A.: A one-dimensional finite element method for simulation-based medical planning for cardiovascular disease. Computer Methods in Biomechanics and Biomedical Engineering 5(3), 195-206 (2002)

20. Warnes, C.A.: ACC/AHA 2008 guidelines for the management of adults with congenital heart disease. Circulation 118(23), 2395-2451 (2008) 